\title{
Marcha de absorção, taxa de acúmulo e exportação de micronutrientes e alumínio pelo tabaco (Nicotiana tabacum L.)
}

\author{
Absorption, rate of accumulation and exporting of micronutrients and aluminum through tobacco \\ (Nicotiana tabacum L.)
}

Marcus Vinicius Cremonesi ${ }^{\star}$, Bruna Ramalho, Priscila Golfetto, Luis Sergio Krepki \& Volnei Pauletti

Universidade Federal do Paraná, Curitiba, PR, Brasil. *Autor para correspondência: marcuscremonesi@gmail.com.

Submissão:13/06/2018 / Aceite: 25/07/2018

\begin{abstract}
RESUMO
O conhecimento da demanda e partição nutricional durante o ciclo pode contribuir significativamente para o manejo adequado da cultura. O presente trabalho objetivou determinar o acúmulo de massa, taxas de absorção e particionamento dos micronutrientes ferro (Fe), manganês $(\mathrm{Mn})$, boro $(\mathrm{B})$, zinco $(\mathrm{Zn})$ e cobre $(\mathrm{Cu})$, além do alumínio (Al), no tabaco (Nicotiana tabacum L.), utilizando uma variedade amplamente cultivada no sul do Brasil. O trabalho foi conduzido em propriedade particular produtora de tabaco localizada no município de Irati, PR, com delineamento experimental em blocos ao acaso com três repetições. Os tratamentos se constituíram de dez épocas de coleta de plantas, em intervalos de 15 dias. Foram avaliados a massa de matéria seca, conteúdo e taxas de acúmulo diárias dos micronutrientes e Al no caule e folhas da planta. Verificou-se que o máximo acúmulo de matéria seca foi próximo aos 68 dias, após o transplantio (DAT), e o pico de acúmulo para a maioria dos micronutrientes ocorreu a partir dos 105 DAT. A ordem de acúmulo de micronutrientes para o tabaco foi $\mathrm{Fe}>\mathrm{Mn}>\mathrm{B}>\mathrm{Zn}>\mathrm{Cu}$ com os valores máximos obtidos aos 105, 135, 135, 135 e 110 DAT, respectivamente, onde mais de $60 \%$ dos micronutrientes absorvidos foram depositados nas folhas. O acúmulo de Al foi de $36 \mathrm{~kg} \mathrm{ha}^{-1}$, com $98,2 \%$ depositado nas folhas.
\end{abstract}

PALAVRAS-CHAVE: solanácea, absorção de nutrientes, exigência nutricional.

\begin{abstract}
Despite the historical and consolidated cultivation of tobacco in Brazil, knowledge of the demand and nutritional partition during the cycle can contribute significantly to the proper management of the crop. The objective of this study was to determine mass accumulation, absorption rates and partitioning of iron ( $\mathrm{Fe})$, manganese $(\mathrm{Mn})$, boron $(\mathrm{B})$, zinc $(\mathrm{Zn})$, copper $(\mathrm{Cu})$ and aluminum (Al) in tobacco (Nicotiana tabacum L.), using a widely cultivated variety in southern Brazil. This study was conducted on a tobacco property located in the municipality of Irati, PR, with a randomized block design with three replicates. The treatments consisted of ten plant collection periods at intervals of 15 days. Dry matter mass, content and daily accumulation rates of micronutrients and $\mathrm{Al}$ in the stem and leaves of the plant were evaluated. It was verified that the maximum accumulation of dry matter mass was close to 68 days after transplanting (DAT), and the peak of accumulation for most micronutrients occurred from 105 DAT. The order of micronutrient accumulation for tobacco was $\mathrm{Fe}>\mathrm{Mn}>\mathrm{B}>\mathrm{Zn}>\mathrm{Cu}$, with maximum values obtained at 105, 135,135, 135 and 110 DAT, respectively, where more than $60 \%$ of the absorbed micronutrients were deposited on the leaves. The accumulation of Al was $36 \mathrm{~kg} \mathrm{ha}^{-1}$, with $98.2 \%$ deposited on sheets.
\end{abstract}

KEYWORDS: solanacea, nutrient absorption, nutritional demand.

\section{INTRODUÇÃO}

O tabaco (Nicotiana tabacum L.) pertence à família Solanaceae, com espécies de alta importância econômica mundial. O Brasil destaca-se como o principal exportador e o terceiro maior produtor mundial de tabaco (RIQUINHO \& HENNINGTON 2016), com uma produção total de 820 mil toneladas na safra de 2018 (IBGE 2018). Cerca de $98 \%$ da produção concentra-se na região Sul, com os três estados produzindo próximo a 799 mil toneladas em 2018 (IBGE 2018). Devido ao alto valor agregado por hectare do produto final, o tabaco tornou-se a principal cultura agrícola não alimentícia (CALDEIRA et al. 2016), sendo utilizado 
inclusive em caldas fitossanitárias para o controle de insetos na agroecologia.

O conhecimento da marcha de absorção, taxa de acúmulo de nutrientes e matéria seca permite definir a exigência dos elementos requeridos em cada estádio fenológico da cultura. Com isso, pode-se estimar a exportação pela colheita e o quanto é necessário retornar ao solo (ECHER et al. 2009, KURTZ et al. 2016), sendo essencial para o manejo da adubação. Os micronutrientes são assim chamados pela baixa quantidade absorvida em relação aos macronutrientes (KIRKBY 2012), porém sua carência afeta o crescimento e a produtividade da planta. As funções dos micronutrientes estão relacionadas à atividade enzimática, catalisação de reações, e demais processos fisiológicos e bioquímicos (DINIZ et al. 1999). Por outro lado, o Al é um elemento não essencial e pode ser tóxico às plantas. Entre os principais efeitos nocivos da alta absorção de Al pelas plantas está a inibição do crescimento e desenvolvimento das raízes (FURLANI et al. 1989), entretanto, não há registros que descrevem seus efeitos na cultura do tabaco.

O tabaco possui um ciclo anual longo, com duração próxima a sete meses (HEEMANN 2009) e permite diversas colheitas durante o ciclo. Pertence à família Solanaceae, composta por espécies que requerem intensa adubação de plantio e cobertura, porém em sua maioria com baixa eficiência na absorção dos nutrientes (SILVA et al. 1994, OLIVEIRA et al. 2009). Sendo assim, devido à escassez de informações sobre a demanda nutricional da planta de tabaco, este estudo teve como objetivo verificar a marcha de absorção e taxas de acúmulo de micronutrientes e de alumínio pela cultura.

\section{MATERIAL E MÉTODOS}

O experimento foi realizado de novembro de 2014 a abril de 2015, em lavoura comercial, localizada no município de Irati, PR. O clima da região, segundo Köppen, é do tipo Cfa (CAVIGLIONE et al. 2000), com temperatura média de $21 \stackrel{\circ}{\circ}$ e pluviosidade acumulada de $970 \mathrm{~mm}$ durante o período do experimento. O solo foi classificado como Latossolo Vermelho distroférrico típico (SANTOS et al. 2013) de textura argilosa, apresentando as seguintes características no local do experimento: 4,08 de $\mathrm{pH}\left(\mathrm{CaCl}_{2}\right)$; 17,9 de $\mathrm{C}\left(\mathrm{mg} \mathrm{dm}^{-3}\right) ; 17,4$ de $\mathrm{P}\left(\mathrm{mg} \mathrm{dm}^{-3}\right) ; 1,66$ de Al; 4,2 de Ca; 1,7 de $\mathrm{Mg}$ e 0,11 de $\mathrm{K}$, todos em $\mathrm{cmol}_{\mathrm{c}} \mathrm{dm}^{-}$ 3; 1,5 de $\mathrm{Cu} ; 59,0$ de $\mathrm{Fe} ; 87,6$ de $\mathrm{Mn}$ e 8,0 de $\mathrm{Zn}$, todos em $\mathrm{mg} \mathrm{kg}^{-1}$. Foi utilizada a aveia preta (Avena strigosa Schreb.) como planta de cobertura no cultivo anterior.

O cultivo do tabaco foi realizado em camalhões, onde as mudas do híbrido Virgínia 405 foram transplantadas em espaçamento de $0,55 \times 1,3 \mathrm{~m}$, totalizando 0,72 planta $\mathrm{m}^{-2}$ ou aproximadamente 14 mil plantas ha-1. A adubação foi realizada antes do transplantio com $50 \mathrm{~kg}$ do formulado $04-14-08$ por 1000 plantas, distribuídos a lanço no sulco de plantio. Aos 70 dias após o transplantio (DAT) foi realizada a capação química das plantas com uso de flumetralina, na dose de $375 \mathrm{~g}$ de ingrediente ativo ha $^{-1}$, aplicados via costal, impedindo seu florescimento. Os demais tratos culturais seguiram de acordo com o realizado habitualmente pelo produtor.

O delineamento utilizado foi em blocos ao acaso com três repetições. Os tratamentos consistiram de dez épocas de coleta da planta inteira, sendo a primeira coleta no dia do transplantio e as próximas realizadas em intervalos de 15 dias. Cada unidade experimental correspondeu a um conjunto de dez plantas aleatoriamente escolhidas e previamente demarcadas, respeitando como bordadura entre elas duas plantas. As folhas senescidas de cada unidade experimental correspondente à época de coleta e das unidades experimentais com coletas futuras foram recolhidas para posteriormente serem acrescentadas a massa seca total das plantas. Após a coleta, as plantas foram separadas em folhas, denominadas fotossinteticamente ativas (FSA) e caule, que após pesagem, foram lavadas com água deionizada e secadas em estufa de circulação forçada de ar a $65^{\circ} \mathrm{C}$, até atingirem massa constante. As amostras tiveram sua massa seca determinada e foram moídas em moinho de bolas e passadas em peneira com malha de 2 $\mathrm{mm}$ para posterior análise química. A determinação dos teores de $\mathrm{Al}, \mathrm{B}, \mathrm{Fe}, \mathrm{Cu}, \mathrm{Mn}$ e $\mathrm{Zn}$ do tecido vegetal deu-se por digestão seca (SILVA 1999) seguido de leitura por espectrometria de emissão atômica por plasma acoplado indutivamente (ICP-AES).

O acúmulo de micronutrientes e alumínio foi calculado considerando a massa seca produzida em cada época e o teor dos elementos para cada parte da planta. Neste caso, a massa seca de folhas resultou da soma das folhas senescidas e FSA.

Os resultados foram analisados por meio de regressão, sendo ajustadas equações significativas a 0,05 de probabilidade pelo teste $F$ com maiores coeficientes de determinação $\left(R^{2}\right)$. Para a determinação das taxas diárias de acúmulo de nutrientes e matéria seca foi utilizada a primeira derivada da equação ajustada para cada elemento (BENINCASA 2003, LIMA et al. 2007, KURTZ et al. 2016). 


\section{RESULTADOS E DISCUSSÃO}

O acúmulo máximo de massa da matéria seca da planta de tabaco foi de $3192,5 \mathrm{~kg} \mathrm{ha}^{-1}$, sendo deste total $1958,5 \mathrm{~kg} \mathrm{ha}^{-1}(61 \%)$ referente à produção de folhas (Figura 1A). Para o caule, o maior acúmulo foi de $1277,1 \mathrm{~kg} \mathrm{ha}^{-1}$ aos 135 (DAT) (Figura 1A). As taxas de acúmulo de matéria seca da planta, folhas e caule foram decrescentes (Figura 1B) e as taxas máximas de acúmulo ocorreram no início do ciclo, correspondendo a 49,3, 30,3 e 18,9 $\mathrm{kg} \mathrm{ha}^{-1}$ no primeiro DAT, respectivamente, para planta, folhas e caule. Os valores passaram a ser negativos a partir de 120 DAT, coincidindo com o momento em que as folhas senescidas superam a FSA em massa seca, indicando perda de massa das folhas após a senescência.

A
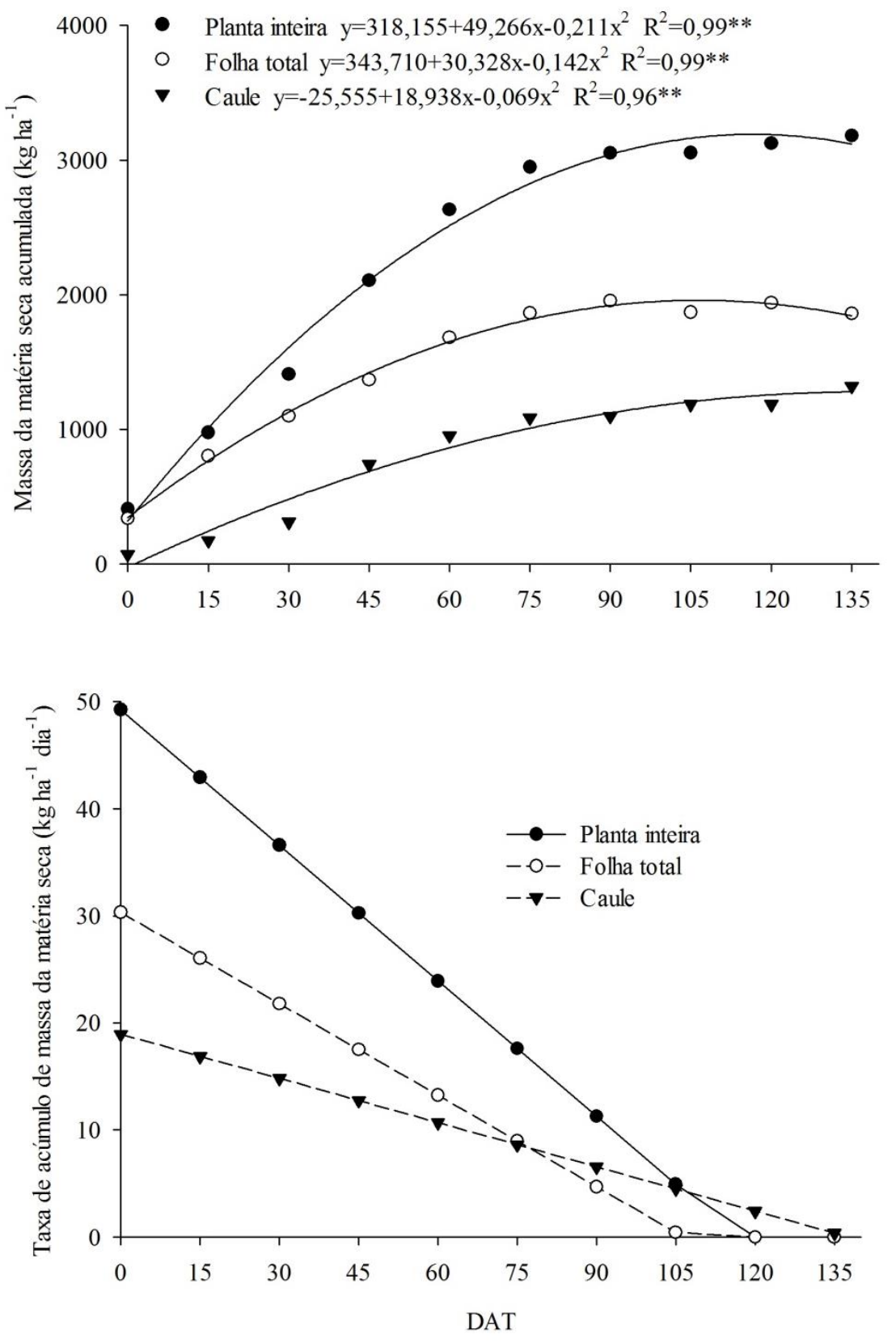

Figura 1. Acúmulo da massa da matéria seca $(A)$ e taxas diárias de acúmulo de massa da matéria seca $(B)$ na planta, folhas e caule de tabaco. ${ }^{* *}$ significativo a $p<0,01$.

Figure 1. Dry matter mass acumulation $(A)$ and daily accumulation rate of dry matter mass $(B)$ in the plant, leaves and tobacco stem. ${ }^{* *}$ significant at $p<0.01$.

O máximo de massa seca de folhas FSA acumulado foi de $1504,5 \mathrm{~kg} \mathrm{ha}^{-1}$ aos 68 DAT (Figura 2). Logo, para o tipo de colheita de planta inteira em que se visa a maior produção de folhas, o período de 65 a 90 DAT é o mais indicado. Após este período ocorre acréscimo das folhas senescidas, que em massa é maior que FSA a partir dos 115 DAT, de modo que a senescência das folhas mais velhas contribui para a redução da massa seca que não é compensada pelo crescimento de novas folhas no ápice. 


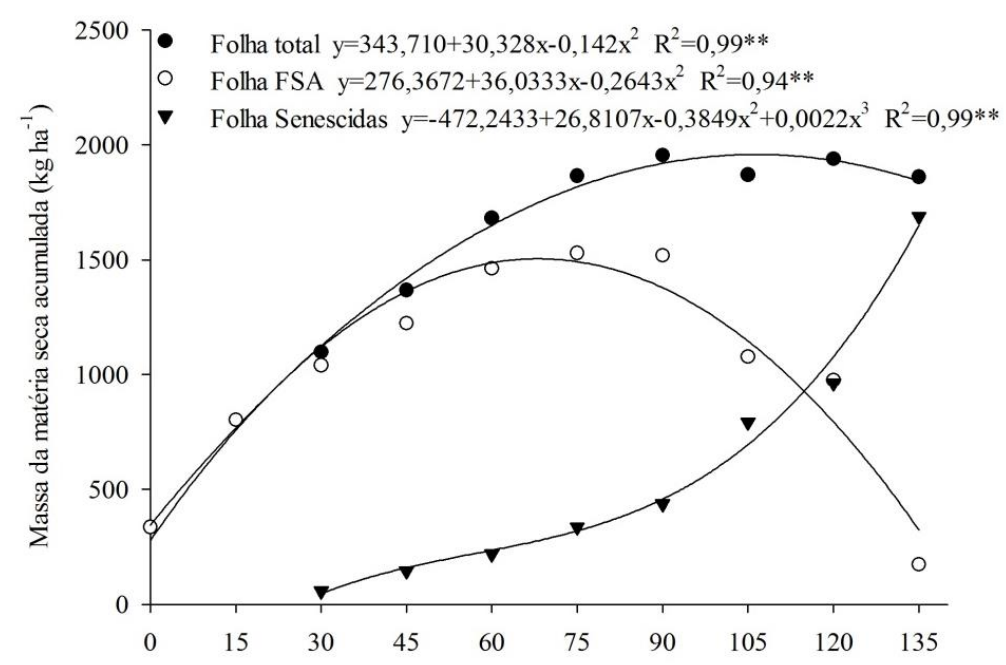

Figura 2. Acúmulo da massa da matéria seca para o total das folhas, folhas fotossinteticamente ativas (FSA) e folhas senescidas do tabaco. ${ }^{* *}$ significativo a $p<0,01$.

Figure 2. Accumulation of dry matter mass for total leaves, photosynthetically active leaves (FSA) and senesced leaves of tobacco. ${ }^{* *}$ significant at $p<0.01$.

A absorção de $\mathrm{Al}$ e micronutrientes pela planta de tabaco dos elementos avaliados teve comportamento similar ao acúmulo de massa da matéria seca, em sua maioria, apresentando acumulação crescente principalmente para as folhas e menor para o caule (Figura 3A). De modo geral, as maiores taxas de acúmulo ocorreram nos primeiros dias após o transplante, com exceção para o Al (Figura 3B) e o Fe que ocorreu após os 50 DAT (Tabela 1).

Tabela 1. Acúmulo, exportação e taxas máximas da massa da matéria seca, alumínio e micronutrientes na planta inteira e folha de tabaco para uma produtividade de $1517 \mathrm{~kg} \mathrm{ha}^{-1}$ para uma população de 14 mil plantas ha-1.

Table 1. Accumulation, exportation and maximum rates of dry matter mass, aluminum and micronutrients in the whole plant, and tobacco leaf for a yield of $1517 \mathrm{~kg} \mathrm{ha}^{-1}$ for a population of 14 thousand plants $h a^{-1}$.

\begin{tabular}{|c|c|c|c|c|c|}
\hline \multirow{3}{*}{ Variável ${ }^{1}$} & \multicolumn{5}{|c|}{ Acúmulo } \\
\hline & \multicolumn{2}{|c|}{------------- Planta ------------ } & \multicolumn{3}{|c|}{----------------- Folha total --------------- } \\
\hline & $\mathrm{kg} \mathrm{ha}^{-1}$ & $\mathrm{DAT}^{2}$ & $\mathrm{~kg} \mathrm{ha}^{-1}$ & $\%$ & DAT \\
\hline MMS & 3192,51 & 120 & 1958,52 & 61,3 & 105 \\
\hline \multirow[t]{2}{*}{ Al } & 36,06 & 110 & 35,87 & 99,5 & 111 \\
\hline & $\mathrm{g} \mathrm{ha}^{-1}$ & DAT & $\mathrm{g} \mathrm{ha}^{-1}$ & $\%$ & DAT \\
\hline $\mathrm{Fe}$ & 22,72 & 105 & 22,32 & 98,2 & 105 \\
\hline Mn & 4,43 & 135 & 3,78 & 85,3 & 135 \\
\hline B & 1,44 & 135 & 1,14 & 79,2 & 135 \\
\hline $\mathrm{Zn}$ & 1,41 & 135 & 0,84 & 59,6 & 135 \\
\hline \multirow[t]{2}{*}{$\mathrm{Cu}$} & 0,16 & 135 & 0,1 & 62,5 & 110 \\
\hline & Exportação & \multicolumn{4}{|c|}{ Taxa máxima de acúmulo } \\
\hline \multirow[t]{2}{*}{ Variável } & Folha total & \multicolumn{2}{|c|}{----------- Planta --------- } & \multicolumn{2}{|c|}{--o--o---- Folha } \\
\hline & $\mathrm{kg} \mathrm{t}^{-1}$ & g planta ${ }^{-1}$ dia $^{-1}$ & DAT & g planta ${ }^{-1}$ dia $^{-1}$ & DAT \\
\hline MMS & - & 49,26 & 0 & 30,31 & 0 \\
\hline \multirow[t]{2}{*}{ Al } & 18,31 & 36,43 & 58 & 36,33 & 59 \\
\hline & $\mathrm{g} \mathrm{t}^{-1}$ & $\mu \mathrm{g}$ planta ${ }^{-1} \mathrm{dia}^{-1}$ & DAT & $\mu \mathrm{g}$ planta ${ }^{-1}$ dia $^{-1}$ & DAT \\
\hline $\mathrm{Fe}$ & 11,4 & 20,4 & 50 & 20,04 & 52 \\
\hline $\mathrm{Mn}$ & 1,93 & 4,03 & 0 & 3,37 & 0 \\
\hline B & 0,58 & 1,71 & 0 & 1,44 & 0 \\
\hline $\mathrm{Zn}$ & 0,43 & 0,99 & 0 & 0,71 & 0 \\
\hline $\mathrm{Cu}$ & 0,05 & 0,4 & 0 & 0,25 & 0 \\
\hline
\end{tabular}



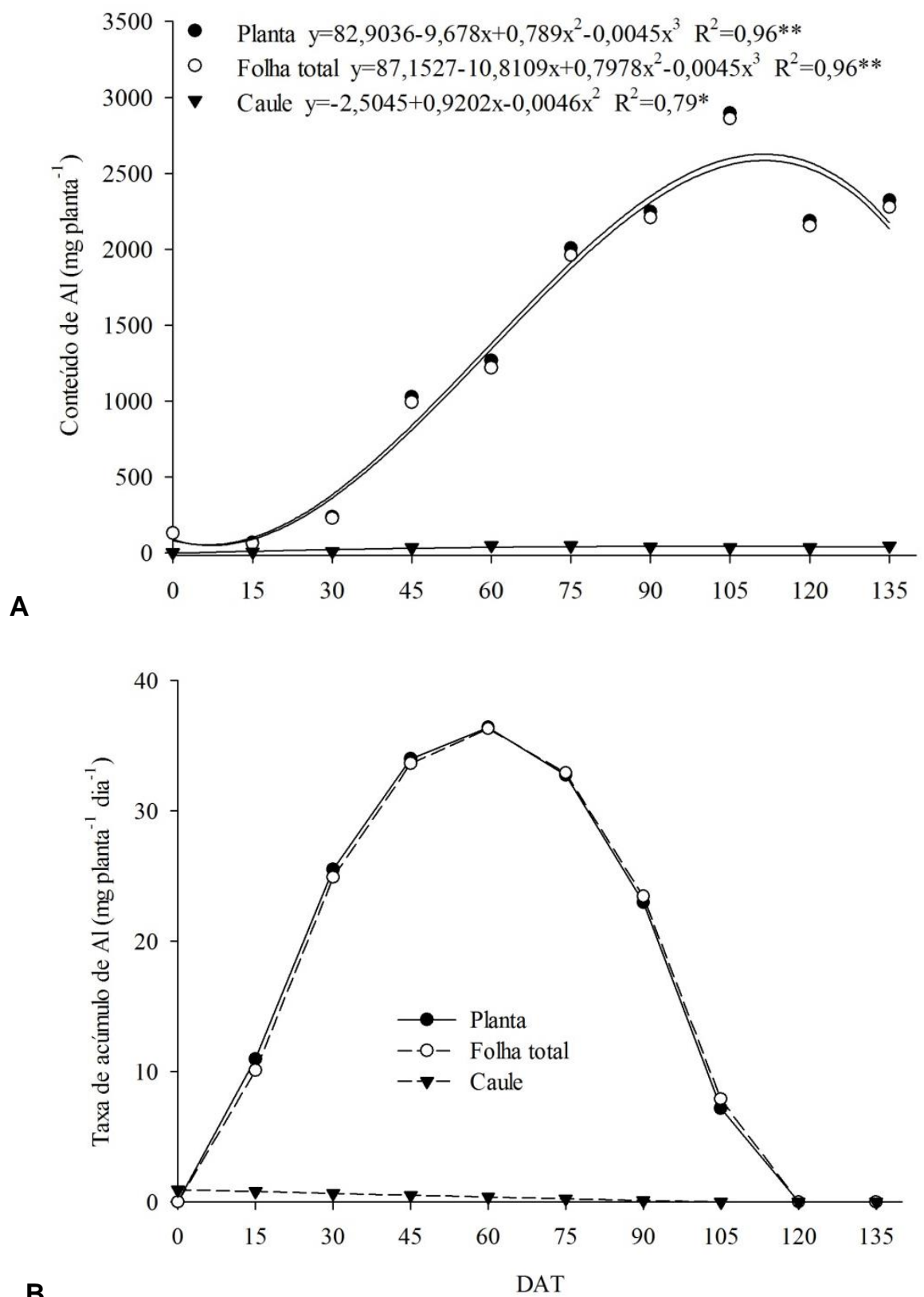

Figura 3. Conteúdo de alumínio (A) e taxas diárias de acúmulo de alumínio (B) na planta, folhas e caule de tabaco. ${ }^{* *} \mathrm{e}^{*}$ : significativo a $\mathrm{p}<0,01$ e $\mathrm{p}<0,05$, respectivamente.

Figure 3. Aluminum content $(A)$ and daily rates of aluminum accumulation $(B)$ in the plant, leaves and tobacco stem. ${ }^{* *}$ and ${ }^{*}$ : significant at $p<0.01$ and $p<0.05$, respectively.

Dentre os elementos analisados, o Al foi o mais absorvido, com máximo de $36,06 \mathrm{~kg} \mathrm{ha}^{-1}$ na planta, sendo $99,5 \%$ depositado nas folhas (Tabela 1). CHENERY (1948) classificou como plantas acumuladoras de Al aquelas cujo valor na massa seca das folhas ultrapasse $1000 \mathrm{mg} \mathrm{kg}^{-1}$. Os teores encontrados para Al chegaram a $2276,5 \mathrm{mg} \mathrm{kg}^{-1}$ nas folhas ao final do ciclo da cultura (Figura 3A), caracterizando-a como acumuladora. A taxa de acúmulo para Al atingiu seu máximo aos 58 DAT para a planta inteira (Figura 3B) com 36,4 $\mathrm{g}_{\text {planta-1 }} \mathrm{dia}^{-1}$, sendo similar para as folhas com 36,3 $\mathrm{g}_{\text {planta }}{ }^{-1} \mathrm{dia}^{-1}$ (Tabela 1).

O solo agrícola deste experimento continha $1,66 \mathrm{cmol}_{\mathrm{c}} \mathrm{dm}^{-3}$ de Al, com saturação por alumínio (m\%) de 14,7, não sendo essa característica suficiente para caracterizá-lo como alítico ou alumínico (SANTOS et al. 2013) e possuía uma saturação por bases (V\%) de 55 na camada de 0 a $20 \mathrm{~cm}$. Estas condições químicas são favoráveis ao desenvolvimento do tabaco (LOPES et al. 1990), porém, disponibiliza Al para absorção pela planta (MOREIRA et al. 2017).

O maior conteúdo de Fe foi observado aos 105 DAT (Figura 4A), em que praticamente todo o Fe da parte aérea do tabaco $\left(1,622 \mathrm{mg}\right.$ planta- $\left.^{-1}\right)$ foi depositado nas folhas $(98,2 \%)$, equivalendo a $22,32 \mathrm{~g} \mathrm{ha}^{-1}$ (Tabela 1). A taxa máxima de acúmulo na planta ocorreu aos 50 DAT, correspondendo a $20,4 \mu \mathrm{g} \mathrm{planta}^{-1}$ 
dia $^{-1}$, decrescendo a partir deste período (Figura 4B). A taxa de acúmulo no caule apresentou comportamento linear decrescente, demonstrando o efeito de diluição deste elemento à medida que a planta se desenvolvia e uma possível translocação para as folhas, atendendo a demanda bioquímica de $\mathrm{Fe}$ no processo de fotossíntese pelo fotossistema I, como ferredoxina (BROADLEY et al. 2012).
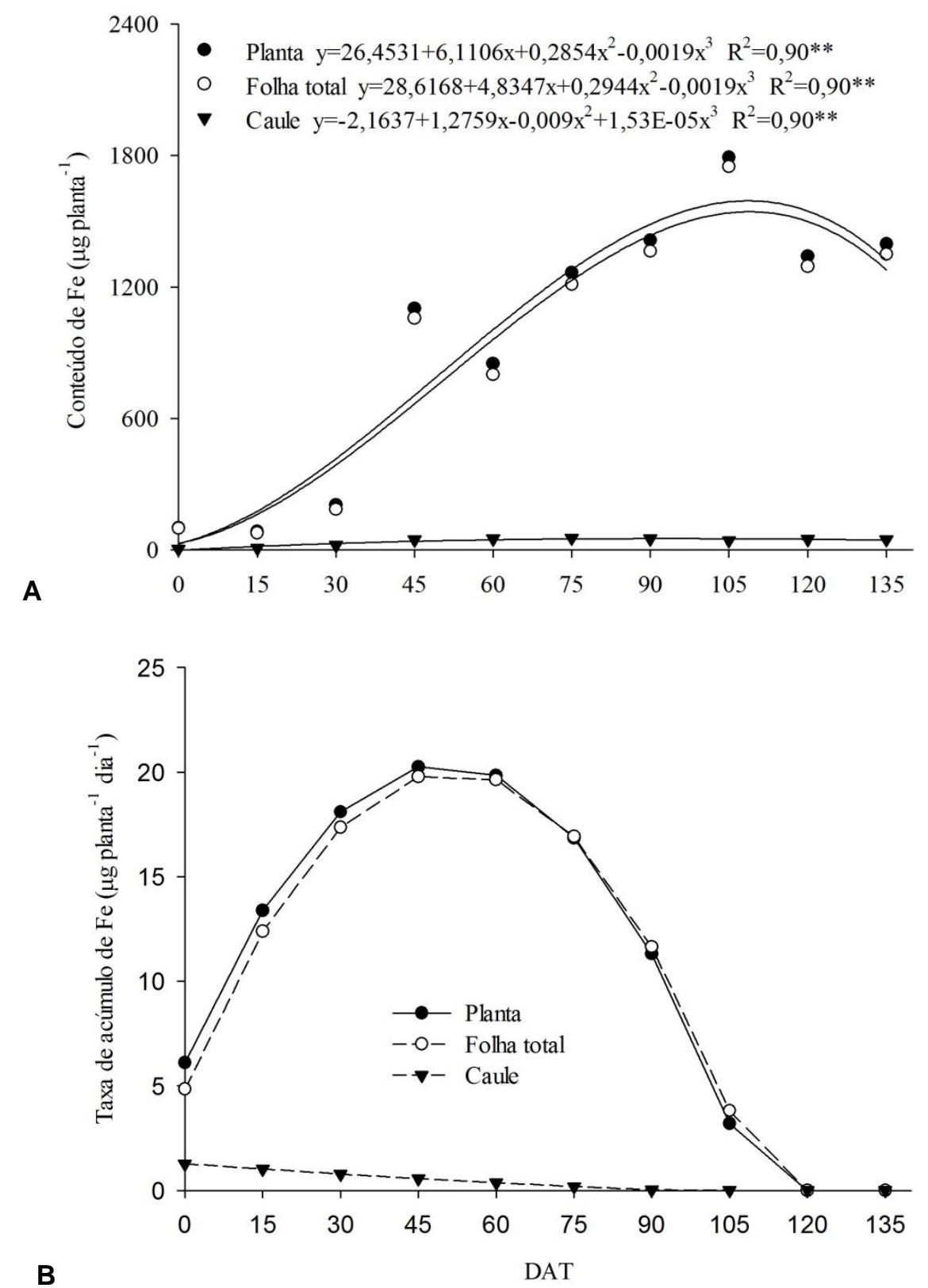

Figura 4. Conteúdo de ferro (A) e taxas diárias de acúmulo de ferro (B) na planta, folhas e caule de tabaco. ** significativo a $p<0,01$.

Figure 4. Iron content $(A)$ and daily rates of iron accumulation $(B)$ in the plant, leaves and tobacco stem. ** significant at $p<0.01$.

Mn e B apresentaram aos 135 DAT acúmulos de $316,4 \mu$ planta $^{-1}$ (Figura 5A) e 102,41 $\mu$ g planta-1 $^{-1}$ (Figura 6A) equivalendo a $4,43 \mathrm{~g} \mathrm{ha}^{-1}$ e $4,14 \mathrm{~g} \mathrm{ha}^{-1}$, respectivamente, ambos depositados majoritariamente nas folhas (Tabela 1). As taxas máximas de acúmulo de $\mathrm{Mn}$ e $\mathrm{B}$ na parte aérea ocorreram no início do ciclo, correspondendo a 4,03 (Figura 5B) e 1,71 $\mathrm{gg}_{\text {glanta }}{ }^{-1} \mathrm{dia}^{-1}$ (Figura 6B), respectivamente. No caule, as taxas de $\mathrm{Mn}$ e $\mathrm{B}$ foram decrescendo com a idade das plantas, com 0,36 e 0,27 $\mu \mathrm{g}_{\text {planta- }}{ }^{-1}$ dia $^{-1}$, respectivamente no início do ciclo (Figuras 5B e 6B).

O Zn apresentou acúmulo quadrático para planta, folha e caule, obtendo um conteúdo de 94,2, 59,9 e 36,1 $\mathrm{\mu g}$ planta- $^{-1}$, respectivamente, aos 135 DAT (Figura 7A). Do total acumulado na planta 59,6\% foi depositado nas folhas, correspondendo a $0,84 \mathrm{~g} \mathrm{ha}^{-1}$ (Tabela 1). As taxas diárias máximas de acúmulo deste elemento ocorreram no início do ciclo para a planta inteira com $0,99 \mu \mathrm{g} \mathrm{planta}^{-1} \mathrm{dia}^{-1}$, folhas com 0,71 $\mu \mathrm{g}$ planta ${ }^{-1} \mathrm{dia}^{-1}$ e caule com $0,28 \mu \mathrm{g}$ planta ${ }^{-1} \mathrm{dia}^{-1}$ (Figura 7B). 

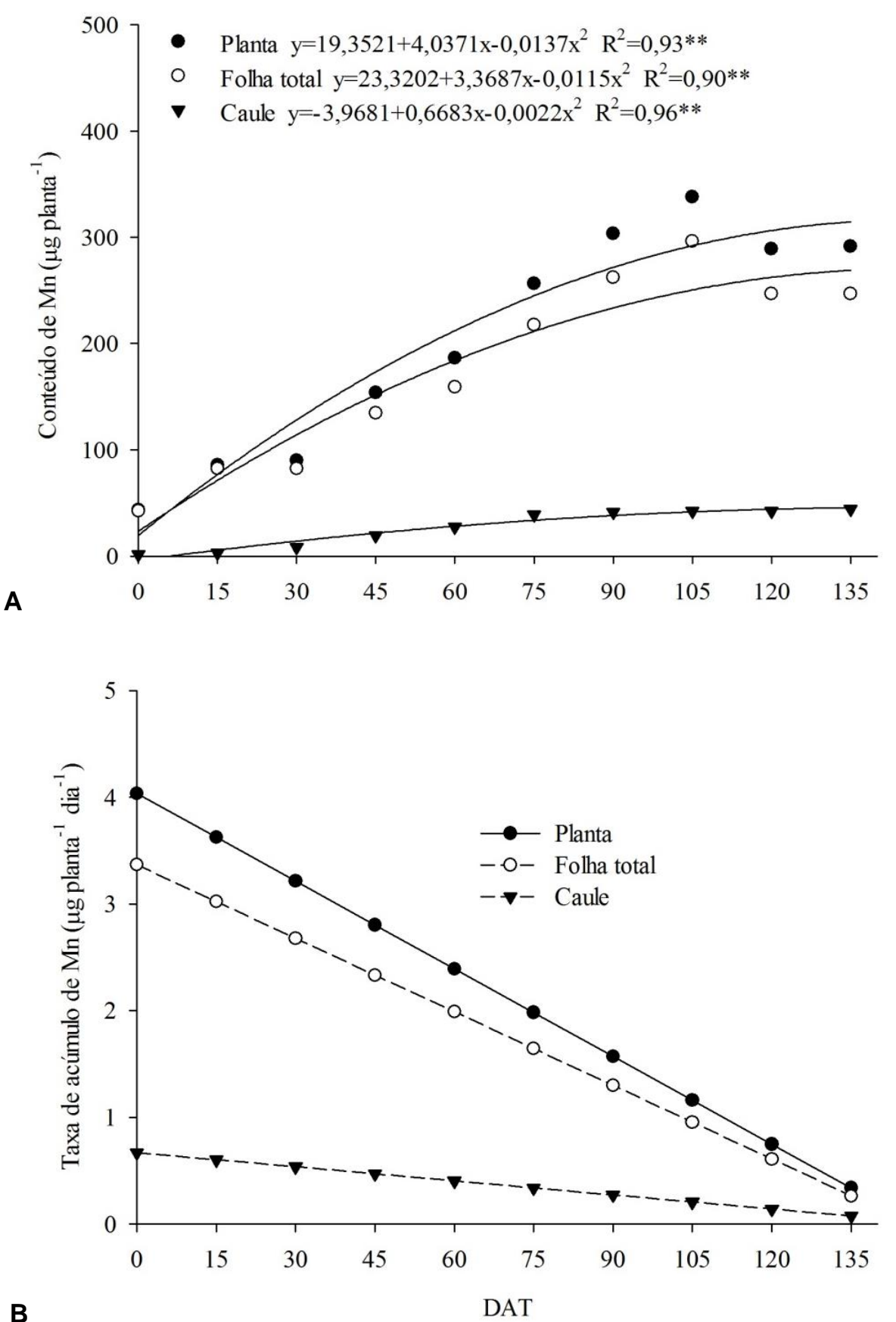

Figura 5. Conteúdo de manganês $(A)$ e taxas diárias de acúmulo de manganês (B) na planta, folhas e caule de tabaco. ${ }^{* *}$ significativo a $\mathrm{p}<0,01$.

Figure 5. Manganese content $(A)$ and daily rates of manganese accumulation $(B)$ in the plant, leaves and tobacco stem. ${ }^{* *}$ significant at $p<0.01$.

A reduzida mobilidade no floema e sua função estrutural de composição da parede celular (BROADLEY et al. 2012) provavelmente favoreceu o maior acúmulo de $\mathrm{B}$ nas folhas em relação ao $\mathrm{Zn}$ e, como consequência, também a maior extração (Tabela 1). Embora culturas da mesma família, como tomate e batata, apresentem teores de $\mathrm{Zn}$ superiores ao de B (MARTINS et al. 2009, SORATTO et al. 2011) possivelmente esse comportamento para a cultura do tabaco seja resultado da capação química, já que o Zn além de cofator enzimático e estabilizador proteico, atua na síntese de auxinas (MENGEL \& KIRKBY 1987, BROADLEY et al. 2012), hormônios relacionados ao crescimento, cuja maior demanda esta relacionada ao meristema da planta (MALTA et al. 2002) e com a remoção do meristema apical ocorre a diminuição da demanda de $\mathrm{Zn}$.

Dentre os micronutrientes avaliados, os menores valores foram encontrados para o $\mathrm{Cu}$, com absorção máxima de 28,16 $\mu$ g planta-1 (Figura 8A), e 62,5\% depositado nas folhas, representando $0,10 \mathrm{~g} \mathrm{ha}^{-1}$ (Tabela 1). A taxa diária de acúmulo foi maior no início do ciclo para a planta, com $0,40 \mu \mathrm{g} \mathrm{planta}^{-1} \mathrm{dia}^{-1}$, folhas com $0,25 \mu \mathrm{g}$ planta ${ }^{-1}$ dia $^{-1}$ e caule com 0,11 0,25 $\mathrm{g} \mathrm{g}$ planta $^{-1}$ dia $^{-1}$ (Figura 8B). 

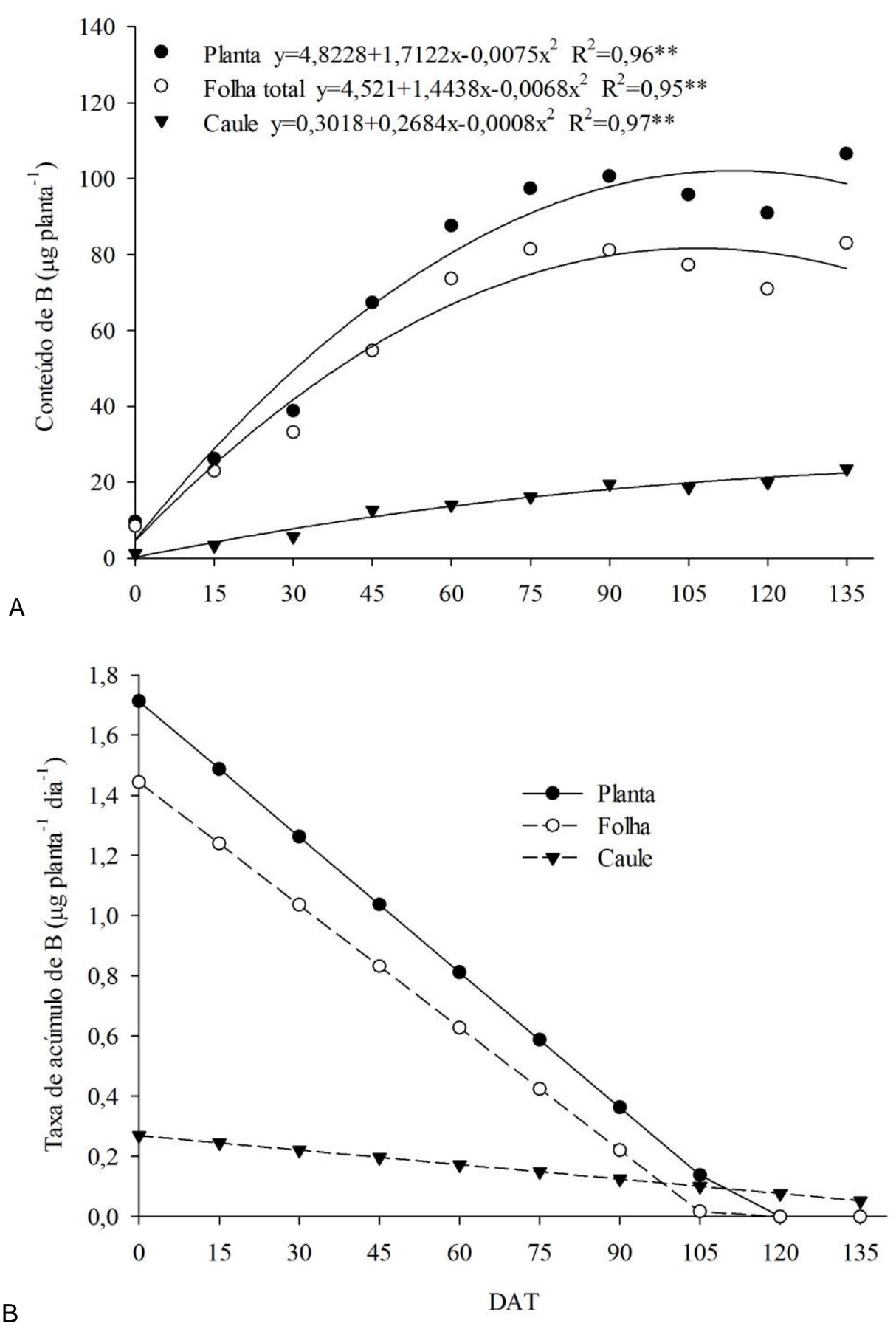

Figura 6. Conteúdo de boro (A) e taxas diárias de acúmulo de boro (B) na planta, folhas e caule de tabaco. ** significativo a $p<0,01$.

Figure 6. Boron content $(A)$ and daily rates of boron accumulation $(B)$ in the plant, leaves and tobacco stem. ${ }^{* *}$ significant at $p<0.01$.

Como a folha de tabaco é o produto comercializado, para cada tonelada de folhas produzidas, as plantas exportaram ao final do ciclo 18,31 $\mathrm{kg}$ de $\mathrm{Al}$ e 11,40, 1,93, 0,58, 0,43 e 0,05 g, respectivamente, de $\mathrm{Fe}, \mathrm{Mn}, \mathrm{B}, \mathrm{Zn}$ e Cu (Tabela 1).

O conteúdo dos micronutrientes $\mathrm{Fe}, \mathrm{Mn}, \mathrm{B}, \mathrm{Zn}$ e Cu são baixos quando comparados aos citados por RODRIGUES et al. (2002) para tomateiro, de 24,64; 48,24; 4,43; 28,35 e 28,12 $\mathrm{mg} \mathrm{planta}^{-1}$ e por SORATTO et al. (2011) para a batata, de 5,$44 ; 1,51 ; 0,14 ; 1,04$ e $0,25 \mathrm{mg}$ planta $^{-1}$, que são espécies da mesma família do tabaco. LUGON-MOULIN et al. (2006), ao avaliarem a concentração de cádmio (Cd) e outros elementos em tabaco para a variedade Virgínia, encontraram valores médios semelhantes aos deste experimento, exceto para $\mathrm{Zn}$ e $\mathrm{Cu}$. O mesmo comportamento foi observado também por VERMA et al. (2010) em diferentes produtos do tabaco. 

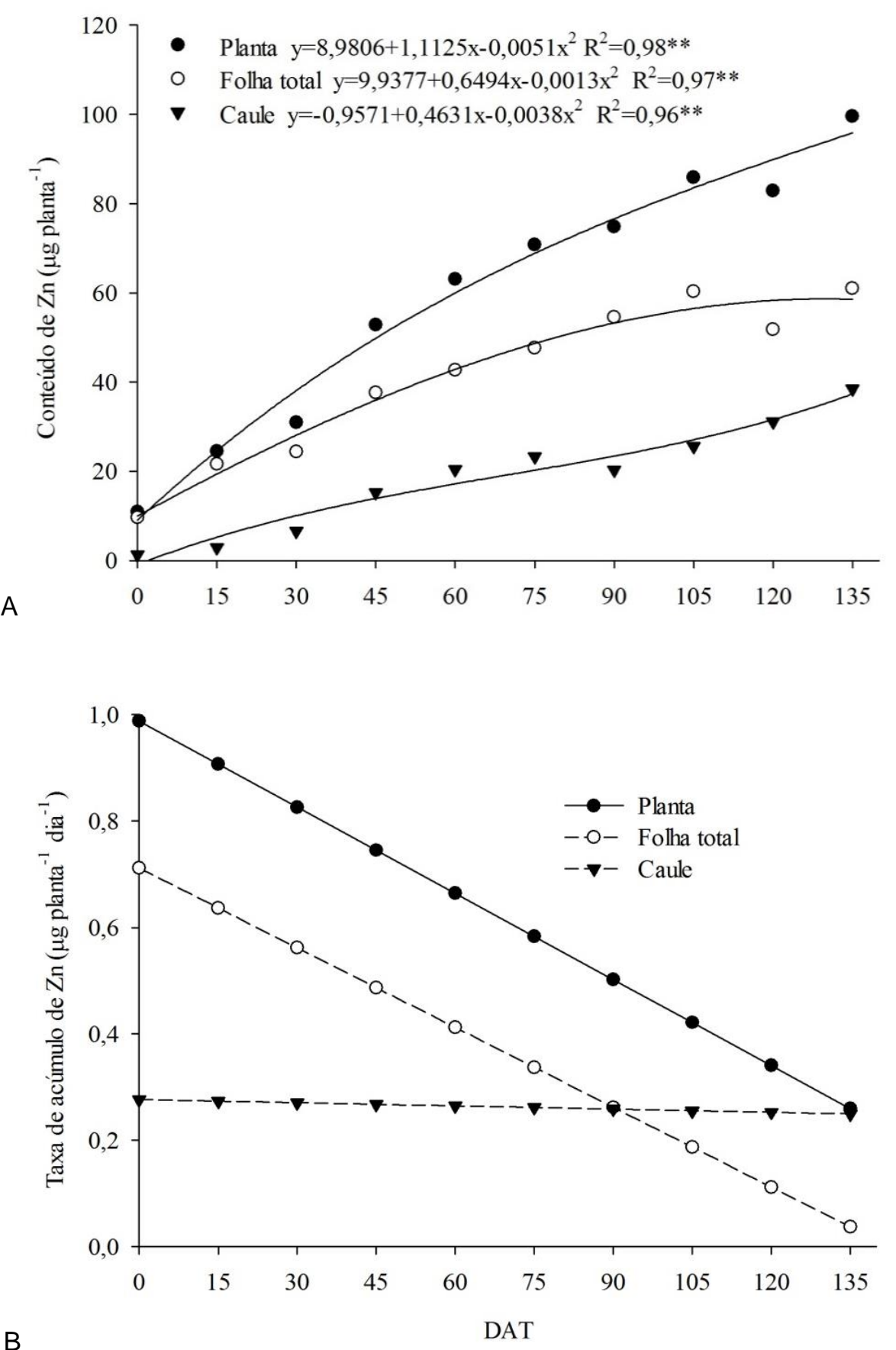

Figura 7. Conteúdo de zinco (A) e taxas diárias de acúmulo de zinco (B) na planta, folhas e caule de tabaco. ${ }^{* *}$ significativo a $p<0,01$.

Figure 7. Zinc content $(A)$ and daily rates of zinc accumulation $(B)$ in the plant, leaves and tobacco stem.

${ }^{* *}$ significant at $p<0.01$.

A ordem de absorção de micronutrientes pela planta de tabaco seguiu a sequência $\mathrm{Fe}>\mathrm{Mn}>\mathrm{B}>\mathrm{Zn}>\mathrm{Cu}$, o mesmo observado para as folhas; e para o caule a ordem foi Fe $>M n>Z n>B>C$. Segundo MOREIRA et al. (2017), para a maioria das gramíneas cultivadas observa-se a sequência de extração $\mathrm{Fe}>M n>Z n>B>C u$ (NAKAGAWA et al. 2009, ZOBIOLE et al. 2011, GLOWACKA 2013) e, para as leguminosas, $\mathrm{Fe}>\mathrm{Zn}>\mathrm{B}>\mathrm{Mn}>\mathrm{Cu}$ (WHALEN et al. 2002, TEIXEIRA et al. 2008). Em olerícolas também são verificadas maiores extrações de $\mathrm{Fe}$ e menores de $\mathrm{Cu}$, com variações entre $\mathrm{Mn}, \mathrm{Zn}$ e $\mathrm{B}$ para cada cultura.

Considerando que as maiores taxas de absorção diárias ocorrem no início do ciclo, o suprimento dos micronutrientes avaliados deve ser garantido até os 45 DAT. Embora a exigência do tabaco não seja tão elevada e não ocorre exportação destes nutrientes em grandes quantidades, é importante atender a necessidade das plantas, evitando a ocorrência de deficiências ou distúrbios fisiológicos proporcionados pela dose e época incorreta de aplicação. 

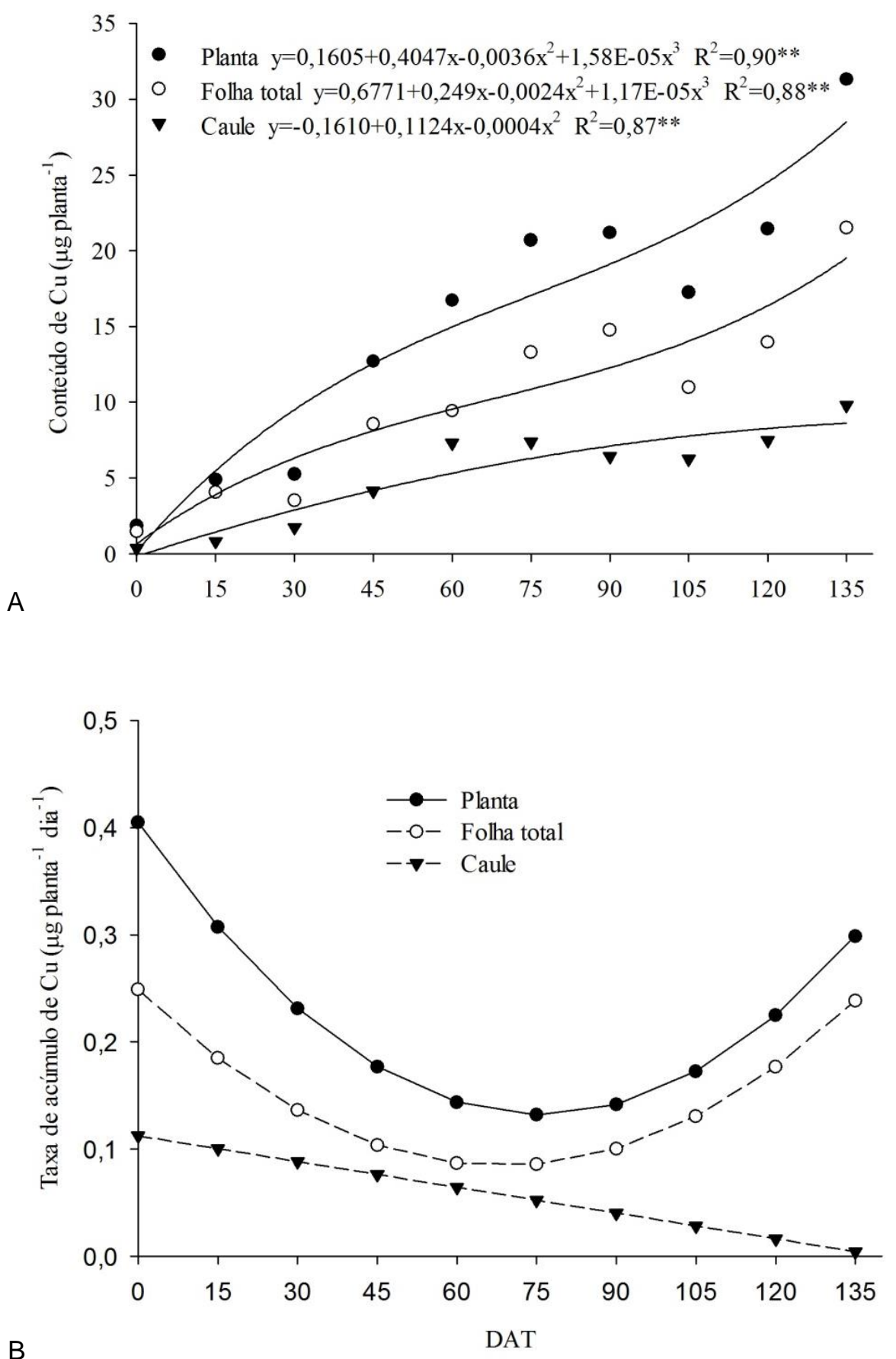

Figura 8. Conteúdo de cobre (A) e taxas diárias de acúmulo de cobre (B) na planta, folhas e caule de tabaco. ${ }^{* *}$ significativo a $\mathrm{p}<0,01$.

Figure 8. Copper content $(A)$ and daily rates of copper accumulation $(B)$ in the plant, leaves and tobacco stem. ${ }^{* *}$ significant at $p<0.01$.

\section{CONCLUSÃO}

O tabaco variedade Virgínia 405 obteve aproximadamente 3,2 $\mathrm{t}$ ha ${ }^{-1}$ de matéria seca ao final do ciclo, com $61,3 \%$ representados pelas folhas.

A taxa máxima de acúmulo de alumínio ocorreu aos 60 dias após o transplantio (DAT), e a maior demanda por micronutrientes no primeiro terço do ciclo da cultura, com exceção do ferro aos 50 DAT.

$O$ acúmulo de micronutrientes em ordem decrescente na planta foi $\mathrm{Fe}>\mathrm{Mn}>\mathrm{B}>\mathrm{Zn}>\mathrm{Cu}$, com 98,2, 85,3, $79,2,59,6$ e 62,5\%, respectivamente, destes nutrientes sendo depositados nas folhas.

\section{REFERÊNCIAS}

BENINCASA MMP. 2003. Análise de crescimento de plantas. 2ed. Jaboticabal: FUNEP. 41p.

BROADLEY M et al. 2012. Functions of nutrients: Micronutrients. IN: MARSCHNER P. (Ed.) Mineral nutrition of higher 
plants. London: Academic Press. p.191-248.

CALDEIRA CM et al. 2016. Qualidade de sementes de tabaco durante o processo de pelotização e armazenamento. Ciência Rural 46: 216-220.

CAVIGLIONE JH et al. 2000. Cartas climáticas do Paraná. Londrina: IAPAR. (CD-Rom).

CHENERY EM 1948. Aluminium in the plant world. Part I. General survey in dieotyledons. Kew Bulletin 3: 173-183.

DINIZ JDN et al. 1999. Absorção de micronutrientes por explantes de bananeira in vitro. Pesquisa agropecuária Brasileira 34: 1385-1391.

ECHER FR et al. 2009. Absorção de nutrientes e distribuição de massa fresca e seca entre órgãos de batata-doce. Horticultura brasileira 27: 176-182.

FURLANI PR et al. 1989. Efeitos fisiológicos do alumínio em plantas. In: Simpósio avançado de solos e nutrição de plantas. Anais. Campinas: Fundação Cargill. p.73-90.

GLOWACKA A. 2013. Uptake of $\mathrm{Cu}, \mathrm{Zn}, \mathrm{Fe}$ and $\mathrm{Mn}$ by maize in the strip cropping system. Plant, Soil and Environment 59: 322-328.

HEEMANN F. 2009. O cultivo do fumo e condições de saúde e segurança dos trabalhadores rurais. Dissertação (Mestrado em Engenharia de Produção). Porto Alegre: UFRGS. 170p.

IBGE/SIDRA. 2018. Levantamento Sistemático da Produção Agrícola: 2018. Disponível em: http://www.sidra.ibge.gov.br/bda/prevsaf/default.asp. Acesso em: 30 mai. 2018.

KIRKBY E. 2012. Introduction, definition, and classification of nutrients. In: MARSCHNER P. (ed.) Mineral nutrition of higher plants. London: Academic Press. p.191-248.

KURTZ C et al. 2016. Crescimento e absorção de nutrientes pela cultivar de cebola Bola Precoce. Horticultura Brasileira 34: $279-288$.

LIMA JF et al. 2007. Índices fisiológicos e crescimento inicial de mamoeiro (Carica papaya L.) em casa de vegetação. Ciência e Agrotecnologia 31: 1358-1363.

LOPES AS et al. 1990. Acidez do solo e calagem. 3.ed. São Paulo: ANDA. 22p. (Boletim técnico 1).

LUGON-MOULIN N et al. 2006. Cadmium concentration in tobacco (Nicotiana tabacum L.) from different countries and its relationship with other elements. Chemosphere 63: 1074-1086.

MALTA MR et al. 2002. Efeito da aplicação de zinco via foliar na síntese de triptofano, aminoácidos e proteínas solúveis em mudas de cafeeiro. Brazilian Journal of Plant Physiology 14: 31-37.

MARTINS CM et al. 2009. Curva de absorção de nutrientes em alface hidropônica. Revista Caatinga 22: 123-128.

MENGEL K \& KIRKBY EA. 1987. Principles of plant nutrition. 4ed. Worblaufen-Bern: International Potash Institute. 687p.

MOREIRA A et al. 2017. Manual de adubação e calagem para o estado do Paraná. Curitiba: SBCS/NEPAR. 482p.

NAKAGAWA $J$ et al. 2009. Teores de nutrientes da folha bandeira e grãos de aveia-preta em função da adubação fosfatada e potássica. Semina 30: 833-840.

OLIVEIRA AR et al. 2009. Absorção de nutrientes e resposta à adubação em linhagens de tomateiro. Horticultura Brasileira 27: 498-504.

RODRIGUES DS et al. 2002. Quantidade absorvida e concentrações de micronutrientes em tomateiro sob cultivo protegido. Scientia Agricola 59: 137-144.

RIQUINHO DL \& HENNINGTON EA. 2016. Sistema integrado de produção do tabaco: saúde, trabalho e condições de vida de trabalhadores rurais no Sul do Brasil. Cadernos de Saúde Pública 32: 1-10.

SANTOS HG et al. 2013. Sistema Brasileiro de Classificação de Solos. 3ed. Brasília: EMBRAPA Solos. 353p.

SILVA JBC et al. 1994. Cultivo do tomate (Lycopersicon esculentum Mill.) para industrialização. Brasília: EMBRAPA CNPH. 36p.

SILVA FC 1999. Manual de análises químicas de solos, plantas e fertilizantes. Brasília: EMBRAPA/CNPTIA. 370p.

SORATTO RP et al. 2011. Extração e exportação de nutrientes em cultivares de batata: II - micronutrientes. Revista Brasileira de Ciência do Solo 35: 2057-2071.

TEIXEIRA CM et al. 2008. Fitomassa, teor e acúmulo de micronutrientes do milheto, feijão-de-porco e guandu-anão, em cultivo solteiro e consorciado. Acta Scientiarum Agronomy 30: 533-538.

VERMA $S$ et al. 2010. Trace metal concentration in different Indian tobacco products and related health implications. Food and Chemical Toxicology 48: 2291-2297.

ZOBIOLE LHS et al. 2011. Sunflower micronutrient uptake curves. Ciência e Agrotecnologia 35: 346-353.

WHALEN JK et al. 2002. Cattle manure and lime amendments to improve crop production of acidic soils in northern Alberta. Canadian Journal of Soil Science 82: 227-238. 